\title{
FORMATIVE AND SUMMATIVE ASSESSMENT IN THE LIGHT OF STUDENTS PERCEPTIONS
}

\author{
Mohammad Ali Chughtai ${ }^{1}$, Mariya Khalid ${ }^{1}$, Saira Afridi ${ }^{1}$, Syed Nasir Shah ${ }^{1}$ \\ 1. Sardar Begum Dental College
}

\section{ABSTRACT}

\section{OBJECTIVES}

To collect information about the Perceptions of dental students regarding Formative and Summative Assessment in Sardar Begum Dental College.

\section{METHODS}

This cross sectional study was conducted at Sardar Begum Dental College, Peshawar. Pre validated Assessment Experience Questionnaire was used to collect information. All the students of $2^{\text {nd }}, 3^{\text {rd }}$, $4^{\text {th }}$ year $B D S$ and House officers who have joined and continued their bachelors education at Sardar Begum Dental College and Hospital from the first year were invited to participate in this study through Convenience sampling technique after taking verbal and written consent.

\section{RESULTS}

Overall response rate was $29.3 \%$ in $2^{\text {nd }}$ Year, $35.1 \%$ in $3^{\text {rd }}$ year, $75.7 \%$ in $4^{\text {th }}$ Year and $92.7 \%$ among house officers.60-70\% students opined that formative assessment experiences stimulated deep learning and were useful in the preparation for summative assessment. Majority of the students were satisfied with the atmosphere, structure and conduct of summative assessment. More than $70 \%$ admitted that they learn more when preparing for summative assessment near the examination. Almost $60 \%$ prefer to have more number of SEQs by claiming that these let them apply deep learning. Despite of accepting the contents of OSCE/OSPE as of good quality, majority students from all the professional years were dissatisfied from the time allocation as well as slides and images placed in each station

\section{CONCLUSION}

Students like formative assessment mainly because of feedback as it helps them in learning as well as preparing for summative assessment. All parts of summative assessment were more satisfactory for the students.

KEY WORDS: Formative, Summative Assessment, Students Perception

\section{INTRODUCTION}

Formative assessment is a process used by teachers and students during instruction that provides feedback to adjust ongoing teaching and learning to improve students' achievement of

\section{Correspondence:}

Dr. Mohammad Ali Chughtai

Sardar Begum Dental College

Contact: 0300-9324492

Email: dentistsaira@gmail.com

https://doi.org/10.37762/jgmds.3-2.34 intended instructional outcomes. ${ }^{1}$ So it is a known fact that the use of Formative assessment (assessment for learning) leads to improvement in quality learning. ${ }^{2}$ In other words teacher engage students in instructional conversations, probe out students thinking by in sighting into it and try to uncover misconceptions in order to correct these. ${ }^{3}$ There are sufficient research evidence available that Formative assessment for learning practices lead to improvement in student learning andachievement. ${ }^{3}$ Critically, the formative assessment process is prospective. It helps to point out the way forward for students, 
and provides the learner with feedback which supports to take steps through the zone of proximal development toward greater levels of autonomy and mastery. ${ }^{1}$

In contrast summative assessments are the assessments of learning. They measure student achievement and progress after a period of learning, and within increasingly broader contexts. They are also used to make judgments about the student learning and to evaluate and hold accountable educational programs. ${ }^{4}$ Results from this assessment may also serve a formative purpose but differ from in a way that they are retrospective regarding teacher moves and student learning. ${ }^{4}$ The tools of summative assessment are designed to measure performance outcomes at the conclusion of a period of learning. ${ }^{4}$ Effectiveness of the curriculum in addition to its design, way of implementation and assessment also depends on perceptions about it by the students. ${ }^{5}$ There are multiple factors like cultural differences and emotions that can affect student's perception about assessment and overall curriculum. ${ }^{6}$ Therefore a wise combination of summative and formative assessment based on learning objectives is required, by taking into consideration student's cultural background, values and implemented education system ${ }^{6}$. This is important because authentic assessment is among the powerful motivators that drive students learning approach ${ }^{6}$. Ultimately positive perception by the students will enhance the students learning motivation. ${ }^{6}$ So it is important to evaluate their perceptions at regular intervals ${ }^{7}$, inorder to keep learning and assessment in interaction.

The importance of evaluation is further highlighted by the drastic change in learning style at school level(strategic learning characterized by motivation to be successful to achieve high grades) to University level Professional education that requires deep learning style which depends on subject interest/understanding and vocational relevance. ${ }^{8}$ Besides this superficial approach to learning by frequently use of old examination material is also a problem in many students. ${ }^{9}$ And particularly medical students are at risk of developing such practice. ${ }^{10}$ Therefore it seems meaningful to evaluate student's perception regarding their study efforts, formative and summative assessments in order to set goals and charting process towards improving learning outcomes. So, aim of this cross sectional study was to collect information about the Perceptions of dental students regarding their study efforts/ practices, Formative and Summative Assessment.

\section{METHODOLOGY}

This cross-sectional study was conducted at Sardar Begum dental college. All the students of 2nd, 3rd, 4th year BDS and House officers who have joined and continued their bachelors education at Sardar Begum Dental College and Hospital from the first year were invited to participate in this study. Non willing participants and those absent at the day of data collection were excluded. Using non-probability convenient sampling technique, pre validated "Assessment Experience Questionnaire", ${ }^{7}$ after being approved by the Advanced Studies and Research board and Ethical Committee of Gandhara University Peshawar was used to collect information..Before finalization of study design permission was taken from the author of Assessment Experience Questionnaire through e-mail. Questionnaire was comprised of three parts; first part comprises of five questions related to Students study efforts. Second part having eight questions related to the Formative assessment, while the third part with fifteen questions belonging to Summative assessment. After taking verbal and written consent they were given to complete the questionnaire.

Data entered and analyzed in Statistical Package for Social Sciences Version 16. For the ease of analysis participants responded to the individual questions as agreed / strongly agreed and disagree / strongly disagree were pooled together as agreed and disagreed respectively. 
Descriptive statistics applied to get frequency of responses by the participants as agreed, disagreed or remain uncertain to the statements of the individual questions. While Chi square test was applied to assess whether there is any significant difference exists between the responses by the participants belonging to different level of Professional education.

\section{RESULTS}

Overall response rate was $29.3 \%$ in 2 nd Year, 35.1\% in 3rd year, $75.7 \%$ in 4 th Year and $92.7 \%$ among house officers.

\section{Part 1: Questions Regarding Study Efforts}

Results from the first part which consists of three questions suggests that most of our students give more time to the studies near their assessment. Result of Question 4 shown, increased trend of assessment driven study among the students with increase in level of professional education. Majority of students disagree to the statement of "being not very keen to obtain position in the class".(Table 1).

Table No 1: Students Perception Regarding Study Efforts

\begin{tabular}{|c|c|c|c|c|c|c|c|c|c|c|c|c|c|}
\hline \multirow{2}{*}{$\begin{array}{l}\text { Questions } \\
\text { Regarding } \\
\text { Study Effort }\end{array}$} & \multicolumn{3}{|c|}{$2^{\text {nd }}$ Year BDS } & \multicolumn{3}{|c|}{$3^{\text {rd }}$ Year BDS } & \multicolumn{3}{|c|}{$4^{\text {th }}$ Year BDS } & \multicolumn{3}{|c|}{ House Officers } & \multirow[t]{2}{*}{ P Value } \\
\hline & A & $\mathbf{U}$ & D & A & $\mathbf{U}$ & D & A & $\mathbf{U}$ & D & A & $\mathbf{U}$ & D & \\
\hline $\begin{array}{l}\text { Q1.I do same } \\
\text { amount of } \\
\text { study each } \\
\text { week } \\
\text { regardless an } \\
\text { assessment } \\
\text { is due or not }\end{array}$ & $\begin{array}{l}4 \\
(2.4 \\
\%)\end{array}$ & $\begin{array}{l}5 \\
(3 \%)\end{array}$ & $\begin{array}{l}13 \\
(7.8 \\
\%)\end{array}$ & $\begin{array}{l}7 \\
(4.2 \%)\end{array}$ & $\begin{array}{l}4 \\
(2.4 \%)\end{array}$ & $\begin{array}{l}15 \\
(9.1 \\
\%)\end{array}$ & $\begin{array}{l}17 \\
(10.3 \\
\%)\end{array}$ & $\begin{array}{l}8 \\
(4.8 \\
\%)\end{array}$ & $\begin{array}{l}28 \\
(17 \%)\end{array}$ & $\begin{array}{l}24 \\
(14.5 \\
\%)\end{array}$ & $\begin{array}{l}20 \\
(12.1 \\
\%)\end{array}$ & $\begin{array}{l}20 \\
(12.1 \\
\%)\end{array}$ & 0.07 \\
\hline $\begin{array}{l}\text { Q2.I can do } \\
\text { well in the } \\
\text { assessment } \\
\text { by doing } \\
\text { selective } \\
\text { study }\end{array}$ & $\begin{array}{l}15 \\
(9.1 \\
\%)\end{array}$ & $\begin{array}{l}2 \\
(1.2 \\
\%)\end{array}$ & $\begin{array}{l}5 \\
(3 \%)\end{array}$ & $\begin{array}{l}8 \\
(4.8 \%)\end{array}$ & $\begin{array}{l}13 \\
(7.8 \%)\end{array}$ & $\begin{array}{l}5 \\
(1.2 \\
\%)\end{array}$ & $\begin{array}{l}35 \\
(21.2 \\
\%)\end{array}$ & $\begin{array}{l}9 \\
(5.4 \\
\%)\end{array}$ & $\begin{array}{l}9 \\
(5.4 \%)\end{array}$ & $\begin{array}{l}44 \\
(26.6 \\
\%)\end{array}$ & $\begin{array}{l}6 \\
(3.6 \%)\end{array}$ & $\begin{array}{l}14 \\
(8.4 \\
\%)\end{array}$ & 0.001 \\
\hline $\begin{array}{l}\text { Q3.In week } \\
\text { when the } \\
\text { assessment } \\
\text { is due, I put } \\
\text { more hours } \\
\text { in study }\end{array}$ & $\begin{array}{l}15 \\
(9.1 \\
\%)\end{array}$ & $\begin{array}{l}2 \\
(1.2 \\
\%)\end{array}$ & $\begin{array}{l}5 \\
(1.2 \\
\%)\end{array}$ & $\begin{array}{l}22 \\
(13.3 \\
\%)\end{array}$ & $\begin{array}{l}2 \\
(1.2 \%)\end{array}$ & $\begin{array}{l}2 \\
(1.2 \\
\%)\end{array}$ & $\begin{array}{l}38 \\
(23 \%)\end{array}$ & $\begin{array}{l}6 \\
(3.6 \\
\%)\end{array}$ & $\begin{array}{l}9 \\
(5.4 \%)\end{array}$ & $\begin{array}{l}46 \\
(27.8 \\
\%)\end{array}$ & $\begin{array}{l}11 \\
(6.6 \%)\end{array}$ & $\begin{array}{l}7 \\
(4.2 \\
\%)\end{array}$ & 0.53 \\
\hline $\begin{array}{l}\text { Q4.I omit to } \\
\text { prepare } \\
\text { subjects } \\
\text { which are } \\
\text { likely to be } \\
\text { minimally } \\
\text { represented } \\
\text { in the } \\
\text { assessment }\end{array}$ & $\begin{array}{l}6 \\
(3.6 \\
\%)\end{array}$ & $\begin{array}{l}10 \\
(6 \%)\end{array}$ & $\begin{array}{l}6 \\
(3.6 \\
\%)\end{array}$ & $\begin{array}{l}12 \\
(12.1 \\
\%)\end{array}$ & $\begin{array}{l}6 \\
(3.6 \%)\end{array}$ & $\begin{array}{l}8 \\
(4.8 \\
\%)\end{array}$ & $\begin{array}{l}26 \\
(15.7 \\
\%)\end{array}$ & $\begin{array}{l}10 \\
(6 \%)\end{array}$ & $\begin{array}{l}17 \\
(10.3 \\
\%)\end{array}$ & $\begin{array}{l}32 \\
(19.3 \\
\%)\end{array}$ & $\begin{array}{l}22 \\
(13.3 \\
\%)\end{array}$ & $\begin{array}{l}10 \\
(6 \%)\end{array}$ & 0.12 \\
\hline $\begin{array}{l}\text { Q5.I am not } \\
\text { very keen on } \\
\text { obtaining } \\
\text { positions in } \\
\text { the class }\end{array}$ & $\begin{array}{l}5 \\
(3 \% \\
)\end{array}$ & $\begin{array}{l}5 \\
(3 \%)\end{array}$ & $\begin{array}{l}12 \\
(12.1 \\
\%)\end{array}$ & $\begin{array}{l}9 \\
(5.4 \%)\end{array}$ & $\begin{array}{l}8 \\
(4.8 \%)\end{array}$ & $\begin{array}{l}9 \\
(5.4 \\
\%)\end{array}$ & $\begin{array}{l}21 \\
(12.7 \\
\%)\end{array}$ & $\begin{array}{l}14 \\
(8.4 \\
\%)\end{array}$ & $\begin{array}{l}18 \\
1(1 \%)\end{array}$ & $\begin{array}{l}28 \\
(17 \%)\end{array}$ & $\begin{array}{l}14 \\
(8.4 \%)\end{array}$ & $\begin{array}{l}22 \\
(13.3 \\
\%)\end{array}$ & 0.56 \\
\hline
\end{tabular}

Part 2: Questions Regarding Formative Assessment 
Overall importance of Formative assessment is well appreciated and admired by the students particularly by the clinical students. About the regularity of feedback provision clinical students of $4^{\text {th }}$ year and house job were more satisfied compared to $2^{\text {nd }}$ and $3^{\text {rd }}$ Professional BDS according to question 3 responses. But students belonging to all professional years accepted the feedback as a source to remedy the mistakes. There was increase trend of reading marks only among the $4^{\text {th }}$ year and House job students compared to $2^{\text {nd }}$ and $3^{\text {rd }}$ yearstudents (Q5). In response to question 6 and 7 majority students accepted the importance of formative assessment in deep learning and its role for the preparation of summative assessment. The numbers of formative assessments are too many were accepted by majority of students of all the professional years (Table 2).

Table No 2: Students Perception Regarding Formative Assessment

\begin{tabular}{|c|c|c|c|c|c|c|c|c|c|c|c|c|c|}
\hline \multirow{2}{*}{$\begin{array}{l}\text { Questions } \\
\text { Regarding } \\
\text { Formative } \\
\text { Assessment }\end{array}$} & \multicolumn{3}{|c|}{$2^{\text {nd }}$ Year BDS } & \multicolumn{3}{|c|}{$3^{\text {rd }}$ Year BDS } & \multicolumn{3}{|c|}{$4^{\text {th }}$ Year BDS } & \multicolumn{3}{|c|}{ House Officers } & \multirow[t]{2}{*}{ P Value } \\
\hline & $\mathrm{A}$ & $\mathrm{U}$ & $\mathrm{D}$ & $\bar{A}$ & $\mathrm{U}$ & $\mathrm{D}$ & A & $\mathrm{U}$ & $\mathrm{D}$ & $A$ & $\bar{U}$ & $\mathrm{D}$ & \\
\hline $\begin{array}{l}\text { Q1.I take } \\
\text { Formative } \\
\text { assessment } \\
\text { regularly and } \\
\text { seriously }\end{array}$ & $\begin{array}{l}17 \\
(10.3 \%)\end{array}$ & $\begin{array}{l}2 \\
(1.2 \%)\end{array}$ & $\begin{array}{l}3 \\
(1.8 \%)\end{array}$ & $\begin{array}{l}15 \\
(9.1 \%)\end{array}$ & $\begin{array}{l}8 \\
(4.8 \%)\end{array}$ & $\begin{array}{l}3 \\
(1.8 \%)\end{array}$ & $\begin{array}{l}37 \\
(22.4 \%)\end{array}$ & $\begin{array}{l}8 \\
(4.8 \%)\end{array}$ & $\begin{array}{l}8 \\
(4.8 \\
\%)\end{array}$ & $\begin{array}{l}50 \\
(30.3 \\
\%)\end{array}$ & $\begin{array}{l}11 \\
(6.6 \\
\%)\end{array}$ & $\begin{array}{l}3 \\
(1.8 \%)\end{array}$ & 0.21 \\
\hline $\begin{array}{l}\text { Q2.Methods } \\
\text { used in } \\
\text { Formative } \\
\text { assessment } \\
\text { are multiple, } \\
\text { diverse and } \\
\text { stimulate } \\
\text { learning }\end{array}$ & $\begin{array}{l}18 \\
(11 \%)\end{array}$ & $\begin{array}{l}3 \\
(1.8 \%)\end{array}$ & $\begin{array}{l}1 \\
(0.6 \%)\end{array}$ & $\begin{array}{l}12 \\
(7.2 \%)\end{array}$ & $\begin{array}{l}9 \\
(5.4 \%)\end{array}$ & $\begin{array}{l}5 \\
(3 \%)\end{array}$ & $\begin{array}{l}39 \\
(23.6 \%)\end{array}$ & $\begin{array}{l}9 \\
(5.4 \%)\end{array}$ & $\begin{array}{l}5 \\
(3 \%)\end{array}$ & $\begin{array}{l}41 \\
(24 \%)\end{array}$ & $\begin{array}{l}13 \\
(7.8 \\
\%)\end{array}$ & $\begin{array}{l}10 \\
(6 \%)\end{array}$ & 0.16 \\
\hline $\begin{array}{l}\text { Q3.Feedbac } \\
\mathrm{k} \text { is regularly } \\
\text { provided by } \\
\text { the Faculty } \\
\text { based on } \\
\text { Formative } \\
\text { assessment }\end{array}$ & $\begin{array}{l}6 \\
(3.6 \%)\end{array}$ & $\begin{array}{l}6 \\
(3.6 \%)\end{array}$ & $\begin{array}{l}10 \\
(6 \%)\end{array}$ & $\begin{array}{l}8 \\
(4.8 \%)\end{array}$ & $\begin{array}{l}9 \\
(5.4 \%)\end{array}$ & $\begin{array}{l}9 \\
(5.4 \%)\end{array}$ & $\begin{array}{l}30 \\
(18.1 \%)\end{array}$ & $\begin{array}{l}14 \\
(14 \%)\end{array}$ & $\begin{array}{l}9 \\
(5.4 \\
\%)\end{array}$ & $\begin{array}{l}32 \\
(19.3 \\
\%)\end{array}$ & $\begin{array}{l}10 \\
(6 \%)\end{array}$ & $\begin{array}{l}22 \\
(13.3 \\
\%)\end{array}$ & 0.04 \\
\hline $\begin{array}{l}\text { Q4.I take the } \\
\text { feedback } \\
\text { seriously to } \\
\text { remedy my } \\
\text { mistakes }\end{array}$ & $\begin{array}{l}14 \\
(8.4 \%)\end{array}$ & $\begin{array}{l}6 \\
(3.6 \%)\end{array}$ & $\begin{array}{l}2 \\
(1.2 \%)\end{array}$ & $\begin{array}{l}16 \\
(9.6 \%)\end{array}$ & $\begin{array}{l}9 \\
(5.4 \%)\end{array}$ & $\begin{array}{l}1 \\
(0.6 \%)\end{array}$ & $\begin{array}{l}37 \\
(22.4 \%)\end{array}$ & $\begin{array}{l}6 \\
(3.6 \%)\end{array}$ & $\begin{array}{l}10 \\
(6 \%)\end{array}$ & $\begin{array}{l}44 \\
(26.6 \\
\%)\end{array}$ & $\begin{array}{l}11 \\
(6.6 \\
\%)\end{array}$ & $\begin{array}{l}9 \\
(5.4 \%)\end{array}$ & 0.15 \\
\hline $\begin{array}{l}\text { Q5.I tend to } \\
\text { read the } \\
\text { marks only }\end{array}$ & $\begin{array}{l}9 \\
(5.4 \%)\end{array}$ & $\begin{array}{l}4 \\
(2.4 \%)\end{array}$ & $\begin{array}{l}9 \\
(5.4 \%)\end{array}$ & $\begin{array}{l}13 \\
(7.8 \%)\end{array}$ & $\begin{array}{l}6 \\
(3.6 \%)\end{array}$ & $\begin{array}{l}7 \\
(4.2 \%)\end{array}$ & $\begin{array}{l}22 \\
(13.3 \%)\end{array}$ & $\begin{array}{l}13 \\
(7.8 \%)\end{array}$ & $\begin{array}{l}18 \\
(11 \%\end{array}$ & $\begin{array}{l}27 \\
(16.3 \\
\%)\end{array}$ & $\begin{array}{l}17 \\
(10.3 \\
\%)\end{array}$ & $\begin{array}{l}20 \\
(12.1 \\
\%)\end{array}$ & 0.95 \\
\hline $\begin{array}{l}\text { Q6.Formativ } \\
\text { e } \\
\text { assessment } \\
\text { is very } \\
\text { helpful in } \\
\text { stimulating } \\
\text { deep } \\
\text { learning }\end{array}$ & $\begin{array}{l}18 \\
(11 \%)\end{array}$ & $\begin{array}{l}2 \\
(1.2 \%)\end{array}$ & $\begin{array}{l}2 \\
(1.2 \%)\end{array}$ & $\begin{array}{l}17 \\
(10.3 \\
\%)\end{array}$ & $\begin{array}{l}5 \\
(3 \%)\end{array}$ & $\begin{array}{l}4 \\
(2.4 \%)\end{array}$ & $\begin{array}{l}38 \\
(23 \%)\end{array}$ & $\begin{array}{l}9 \\
(5.4 \%)\end{array}$ & $\begin{array}{l}6 \\
(3.6 \\
\%)\end{array}$ & $\begin{array}{l}50 \\
(30.3 \\
\%)\end{array}$ & $\begin{array}{l}9 \\
(5.4 \\
\%)\end{array}$ & $\begin{array}{l}5 \\
(3 \%)\end{array}$ & 0.84 \\
\hline $\begin{array}{l}\text { Q7.Formativ } \\
\text { e } \\
\text { assessment } \\
\text { is very } \\
\text { helpful in } \\
\text { preparing for } \\
\text { Summative } \\
\text { assessment }\end{array}$ & $\begin{array}{l}19 \\
(11.5 \%)\end{array}$ & $\begin{array}{l}1 \\
(0.6 \%)\end{array}$ & $\begin{array}{l}2 \\
(1.2 \%)\end{array}$ & $\begin{array}{l}16 \\
(9.6 \%)\end{array}$ & $\begin{array}{l}9 \\
(5.4 \%)\end{array}$ & $\begin{array}{l}1 \\
(0.6 \%)\end{array}$ & $\begin{array}{l}41 \\
(24 \%)\end{array}$ & $\begin{array}{l}9 \\
(5.4 \%)\end{array}$ & $\begin{array}{l}3 \\
(1.8 \\
\%)\end{array}$ & $\begin{array}{l}54 \\
(32.7 \\
\%)\end{array}$ & $\begin{array}{l}6 \\
(3.6 \\
\%)\end{array}$ & $\begin{array}{l}4 \\
(2.4 \%)\end{array}$ & 0.17 \\
\hline $\begin{array}{l}\text { Q8. There are } \\
\text { too many } \\
\text { Formative } \\
\text { assessments } \\
\text { in a year }\end{array}$ & $\begin{array}{l}18 \\
(11 \%)\end{array}$ & $\begin{array}{l}2 \\
(1.2 \%)\end{array}$ & $\begin{array}{l}2 \\
(1.2 \%)\end{array}$ & $\begin{array}{l}13 \\
(7.8 \%)\end{array}$ & $\begin{array}{l}5 \\
(3 \%)\end{array}$ & $\begin{array}{l}8 \\
(4.8 \%)\end{array}$ & $\begin{array}{l}29 \\
(17.5 \%)\end{array}$ & $\begin{array}{l}15 \\
(9.1 \%)\end{array}$ & $\begin{array}{l}9 \\
(5.4 \\
\%)\end{array}$ & $\begin{array}{l}27 \\
(16.3 \\
\%)\end{array}$ & $\begin{array}{l}18 \\
(11 \%\end{array}$ & $\begin{array}{l}19 \\
(11.5 \\
\%)\end{array}$ & 0.04 \\
\hline
\end{tabular}




\section{Part 3: Questions Regarding Summative Assessment}

This section primarily evaluated about the summative assessments. Majority of students agreed to the statement that there are too many summative assessments in a year. In reply to question 2 and 3, majority students from all the professional years admired that the weightage of curriculum based assessment is appropriate and shown satisfaction about the examination environment and conduct. Regarding exam format in question 4, 5 and 6, majority students from all the professional years accepted the good correlation of examination content with the learning objectives, ease of understanding the questions and number of MCQs are adequate regarding the subject matter. Most students were in favor of different other types of MCQs besides one best type (Q7). In reply to question 8 again majority students prefer to have more number of SEQs by claiming that these let them apply deep learning. Despite of accepting the contents of OSCE/OSPE as of good quality, majority students from all the professional years were dissatisfied from the time allocation as well as slides and images placed in each station (Q 9, 10, 11).

Majority of students accepted that the examination invigilators and observers were impartial and there was hardly any room for unfair means $(\mathrm{Q} 12,13)$. Another appreciable finding regarding our assessment was that maximum students accepted that, they learn new things or gain new knowledge while preparing for the assessment (Q14). But, most of them were of opinion that they tend to forget some of the studied stuff after assessment (Q15)(Table 3).

Table No 3: Students Perception Regarding Summative Assessment

\begin{tabular}{|c|c|c|c|c|c|c|c|c|c|c|c|c|c|}
\hline \multirow{2}{*}{$\begin{array}{l}\text { Questions } \\
\text { Regarding } \\
\text { Summative } \\
\text { Assessment }\end{array}$} & \multicolumn{3}{|c|}{$2^{\text {nd }}$ Year BDS } & \multicolumn{3}{|c|}{$3^{\text {rd }}$ Year BDS } & \multicolumn{3}{|c|}{$4^{\text {th }}$ Year BDS } & \multicolumn{3}{|c|}{ House Officers } & \multirow{2}{*}{$\begin{array}{l}\mathrm{P} \\
\text { Value }\end{array}$} \\
\hline & A & $\mathbf{U}$ & D & A & $\mathbf{U}$ & D & $\mathbf{A}$ & $\mathbf{U}$ & D & A & U & D & \\
\hline $\begin{array}{l}\text { Q1.There are } \\
\text { too many } \\
\text { Summative } \\
\text { Assessment in } \\
\text { a year }\end{array}$ & $\begin{array}{l}18 \\
(11 \% \\
)\end{array}$ & $\begin{array}{l}2 \\
(1.2 \\
\%)\end{array}$ & $\begin{array}{l}2 \\
(1.2 \%)\end{array}$ & $\begin{array}{l}16 \\
(9.6 \\
\%)\end{array}$ & $\begin{array}{l}2 \\
(1.2 \\
\%)\end{array}$ & $\begin{array}{l}8 \\
(4.8 \\
\%)\end{array}$ & $\begin{array}{l}30 \\
(18.1 \\
\%)\end{array}$ & $\begin{array}{l}10 \\
(6 \%)\end{array}$ & $\begin{array}{l}13 \\
(7.8 \\
\%)\end{array}$ & $\begin{array}{l}29 \\
(17.5 \%)\end{array}$ & $\begin{array}{l}10 \\
(6 \%)\end{array}$ & $\begin{array}{l}25 \\
(15 . \\
1 \%)\end{array}$ & 0.07 \\
\hline $\begin{array}{l}\text { Q2.Weightage } \\
\text { of Curriculum } \\
\text { based } \\
\text { Assessment is } \\
\text { appropriate in } \\
\text { professional } \\
\text { exam and result }\end{array}$ & $\begin{array}{l}11 \\
(6.6 \\
\%)\end{array}$ & $\begin{array}{l}10 \\
(6 \%)\end{array}$ & $\begin{array}{l}1 \\
(0.6 \%)\end{array}$ & $\begin{array}{l}13 \\
(7.8 \\
\%)\end{array}$ & $\begin{array}{l}9 \\
(5.4 \\
\%)\end{array}$ & $\begin{array}{l}4 \\
(2.4 \\
\%)\end{array}$ & $\begin{array}{l}30 \\
(18.1 \\
\%)\end{array}$ & $\begin{array}{l}19 \\
(11.5 \\
\%)\end{array}$ & $\begin{array}{l}4 \\
(2.4 \\
\%)\end{array}$ & $\begin{array}{l}37 \\
(23.6 \%)\end{array}$ & $\begin{array}{l}17 \\
(10.3 \\
\%)\end{array}$ & $\begin{array}{l}10 \\
(6 \% \\
)\end{array}$ & 0.51 \\
\hline $\begin{array}{l}\text { Q3.General } \\
\text { atmosphere } \\
\text { and conduct of } \\
\text { examination is } \\
\text { satisfactory }\end{array}$ & $\begin{array}{l}19 \\
(11.5 \\
\%)\end{array}$ & $\begin{array}{l}1 \\
(0.6 \\
\%)\end{array}$ & $\begin{array}{l}2 \\
(1.2 \%)\end{array}$ & $\begin{array}{l}14 \\
(14 \% \\
)\end{array}$ & $\begin{array}{l}4 \\
(2.4 \\
\%)\end{array}$ & $\begin{array}{l}8 \\
(4.8 \\
\%)\end{array}$ & $\begin{array}{l}41 \\
(24 \%)\end{array}$ & $\begin{array}{l}6 \\
(3.6 \\
\%)\end{array}$ & $\begin{array}{l}6 \\
(3.6 \\
\%)\end{array}$ & $\begin{array}{l}40 \\
(24.2 \%)\end{array}$ & $\begin{array}{l}7 \\
(4.2 \%)\end{array}$ & $\begin{array}{l}17 \\
(10 . \\
3 \%)\end{array}$ & 0.11 \\
\hline $\begin{array}{l}\text { Q4.Contents of } \\
\text { the examination } \\
\text { correlate well } \\
\text { with the } \\
\text { Learning } \\
\text { objectives }\end{array}$ & $\begin{array}{l}19 \\
(11.5 \\
\%)\end{array}$ & $\begin{array}{l}1 \\
(0.6 \\
\%)\end{array}$ & $\begin{array}{l}2 \\
(1.2 \%)\end{array}$ & $\begin{array}{l}10 \\
(6 \%)\end{array}$ & $\begin{array}{l}10 \\
(6 \%)\end{array}$ & $\begin{array}{l}6 \\
(3.6 \\
\%)\end{array}$ & $\begin{array}{l}42 \\
(24.4 \\
\%)\end{array}$ & $\begin{array}{l}2 \\
(1.2 \\
\%)\end{array}$ & $\begin{array}{l}9 \\
(5.4 \\
\%)\end{array}$ & $\begin{array}{l}46 \\
(27.8 \%)\end{array}$ & $\begin{array}{l}11 \\
(6.6 \%)\end{array}$ & $\begin{array}{l}7 \\
(4.2 \\
\%)\end{array}$ & 0.001 \\
\hline $\begin{array}{lr}\text { Q5.The } & \\
\text { questions are } \\
\text { easy } & \text { to } \\
\text { understand } & \end{array}$ & $\begin{array}{l}14 \\
(8.4 \\
\%)\end{array}$ & $\begin{array}{l}4 \\
(2.4 \\
\%)\end{array}$ & $\begin{array}{l}4 \\
(2.4 \%)\end{array}$ & $\begin{array}{l}16 \\
(9.6 \\
\%)\end{array}$ & $\begin{array}{l}2 \\
(1.2 \\
\%)\end{array}$ & $\begin{array}{l}8 \\
(4.8 \\
\%)\end{array}$ & $\begin{array}{l}28 \\
(17 \%)\end{array}$ & $\begin{array}{l}9 \\
(5.4 \\
\%)\end{array}$ & $\begin{array}{l}16 \\
(9.6 \\
\%)\end{array}$ & $\begin{array}{l}42 \\
(25.4 \%)\end{array}$ & $\begin{array}{l}15 \\
(9.1 \%)\end{array}$ & $\begin{array}{l}7 \\
(4.2 \\
\%)\end{array}$ & 0.13 \\
\hline $\begin{array}{l}\text { Q6.There are } \\
\text { adequate } \\
\text { number of } \\
\text { MCQs for each } \\
\text { module and }\end{array}$ & $\begin{array}{l}20 \\
(12.1 \\
\%)\end{array}$ & $\begin{array}{l}0 \\
(0 \%)\end{array}$ & $\begin{array}{l}2 \\
(1.2 \%)\end{array}$ & $\begin{array}{l}17 \\
(10.3 \\
\%)\end{array}$ & $\begin{array}{l}3 \\
(1.8 \\
\%)\end{array}$ & $\begin{array}{l}6 \\
(3.6 \\
\%)\end{array}$ & $\begin{array}{l}33 \\
(20 \%)\end{array}$ & $\begin{array}{l}10 \\
(6 \%)\end{array}$ & $\begin{array}{l}10 \\
(6 \%)\end{array}$ & $\begin{array}{l}37 \\
(22.4 \%)\end{array}$ & $\begin{array}{l}8 \\
(4.8 \%)\end{array}$ & $\begin{array}{l}19 \\
(11 . \\
5 \%)\end{array}$ & 0.09 \\
\hline
\end{tabular}




\begin{tabular}{|c|c|c|c|c|c|c|c|c|c|c|c|c|c|}
\hline $\begin{array}{l}\text { subject in a } \\
\text { paper }\end{array}$ & & & & & & & & & & & & & \\
\hline $\begin{array}{l}\text { Q7.There } \\
\text { should be many } \\
\text { types of MCQs } \\
\text { in addition to } \\
\text { one best/ } \\
\text { correct type }\end{array}$ & $\begin{array}{l}8 \\
(4.8 \\
\%)\end{array}$ & $\begin{array}{l}4 \\
(2.4 \\
\%)\end{array}$ & $\begin{array}{l}10 \\
(6 \%)\end{array}$ & $\begin{array}{l}17 \\
(10.3 \\
\%)\end{array}$ & $\begin{array}{l}6 \\
(3.6 \\
\%)\end{array}$ & $\begin{array}{l}3 \\
(1.8 \\
\%)\end{array}$ & $\begin{array}{l}29 \\
(17.5 \\
\%)\end{array}$ & $\begin{array}{l}15 \\
(9.1 \\
\%)\end{array}$ & $\begin{array}{l}9 \\
(5.4 \\
\%)\end{array}$ & $\begin{array}{l}41 \\
(24 \%)\end{array}$ & $\begin{array}{l}14 \\
(14 \%)\end{array}$ & $\begin{array}{l}9 \\
(5.4 \\
\%)\end{array}$ & 0.03 \\
\hline $\begin{array}{l}\text { Q8.There } \\
\text { should be } \\
\text { SEQs in the } \\
\text { assessment as } \\
\text { these let us } \\
\text { apply deep } \\
\text { learning }\end{array}$ & $\begin{array}{l}17 \\
(10.3 \\
\%)\end{array}$ & $\begin{array}{l}2 \\
(1.2 \\
\%)\end{array}$ & $\begin{array}{l}3 \\
(1.8 \%)\end{array}$ & $\begin{array}{l}18 \\
(11 \% \\
)\end{array}$ & $\begin{array}{l}4 \\
(2.4 \\
\%)\end{array}$ & $\begin{array}{l}4 \\
(2.4 \\
\%)\end{array}$ & $\begin{array}{l}36 \\
(21.8 \\
\%)\end{array}$ & $\begin{array}{l}11 \\
(6.6 \\
\%)\end{array}$ & $\begin{array}{l}6 \\
(3.6 \\
\%)\end{array}$ & $\begin{array}{l}53 \\
(32.1 \%)\end{array}$ & $\begin{array}{l}6 \\
(3.6 \%)\end{array}$ & $\begin{array}{l}5 \\
(3 \% \\
)\end{array}$ & 0.50 \\
\hline $\begin{array}{l}\text { Q9.The } \\
\text { contents of } \\
\text { OSPE/ OSCE } \\
\text { are of good } \\
\text { quality }\end{array}$ & $\begin{array}{l}19 \\
(11.5 \\
\%)\end{array}$ & $\begin{array}{l}2 \\
(1.2 \\
\%)\end{array}$ & $\begin{array}{l}1 \\
(0.6 \%)\end{array}$ & $\begin{array}{l}16 \\
(9.6 \\
\%)\end{array}$ & $\begin{array}{l}6 \\
(3.6 \\
\%)\end{array}$ & $\begin{array}{l}4 \\
(2.4 \\
\%)\end{array}$ & $\begin{array}{l}37 \\
(22.4 \\
\%)\end{array}$ & $\begin{array}{l}6 \\
(3.6 \\
\%)\end{array}$ & $\begin{array}{l}10 \\
(6 \%)\end{array}$ & $\begin{array}{l}52 \\
(31.5 \%)\end{array}$ & $\begin{array}{l}7 \\
(4.2 \%)\end{array}$ & $\begin{array}{l}5 \\
(3 \% \\
)\end{array}$ & 0.20 \\
\hline $\begin{array}{l}\text { Q10.The time } \\
\text { allotted for each } \\
\text { station is } \\
\text { adequate for } \\
\text { the response }\end{array}$ & $\begin{array}{l}5 \\
(3 \%)\end{array}$ & $\begin{array}{l}1 \\
(0.6 \\
\%)\end{array}$ & $\begin{array}{l}16 \\
(9.6 \%)\end{array}$ & $\begin{array}{l}8 \\
(4.8 \\
\%)\end{array}$ & $\begin{array}{l}6 \\
(3.6 \\
\%)\end{array}$ & $\begin{array}{l}12 \\
(7.2 \\
\%)\end{array}$ & $\begin{array}{l}15 \\
(9.1 \%)\end{array}$ & $\begin{array}{l}6 \\
(3.6 \\
\%)\end{array}$ & $\begin{array}{l}32 \\
(19.3 \\
\%)\end{array}$ & $\begin{array}{l}26 \\
(15.7 \%)\end{array}$ & $\begin{array}{l}11 \\
(6.6 \%)\end{array}$ & $\begin{array}{l}27 \\
(16 . \\
3 \%)\end{array}$ & 0.14 \\
\hline $\begin{array}{l}\text { Q11.The slides } \\
\text { and images } \\
\text { placed on } \\
\text { OSPE/OSCE } \\
\text { are of good } \\
\text { quality }\end{array}$ & $\begin{array}{l}10 \\
(6 \%)\end{array}$ & $\begin{array}{l}1 \\
(0.6 \\
\%)\end{array}$ & $\begin{array}{l}11 \\
(6.6 \%)\end{array}$ & $\begin{array}{l}5 \\
(3 \%)\end{array}$ & $\begin{array}{l}4 \\
(2.4 \\
\%)\end{array}$ & $\begin{array}{l}17 \\
(10.3 \\
\%)\end{array}$ & $\begin{array}{l}20 \\
(12.1 \\
\%)\end{array}$ & $\begin{array}{l}10 \\
(6 \%)\end{array}$ & $\begin{array}{l}23 \\
(14 \% \\
)\end{array}$ & $\begin{array}{l}33 \\
(20 \%)\end{array}$ & $\begin{array}{l}11 \\
(6.6 \%)\end{array}$ & $\begin{array}{l}20 \\
(12 . \\
1 \%)\end{array}$ & 0.05 \\
\hline $\begin{array}{l}\text { Q12.The } \\
\text { observers and } \\
\text { invigilators are } \\
\text { impartial }\end{array}$ & $\begin{array}{l}6 \\
(3.6 \\
\%)\end{array}$ & $\begin{array}{l}9 \\
(5.4 \\
\%)\end{array}$ & $\begin{array}{l}7 \\
(4.2 \%)\end{array}$ & $\begin{array}{l}12 \\
(7.2 \\
\%)\end{array}$ & $\begin{array}{l}8 \\
(4.8 \\
\%)\end{array}$ & $\begin{array}{l}6 \\
(3.6 \\
\%)\end{array}$ & $\begin{array}{l}26 \\
(15.7 \\
\%)\end{array}$ & $\begin{array}{l}17 \\
(10.3 \\
\%)\end{array}$ & $\begin{array}{l}10 \\
(6 \%)\end{array}$ & $\begin{array}{l}32 \\
(19.3 \%)\end{array}$ & $\begin{array}{l}15 \\
(9.1 \%)\end{array}$ & $\begin{array}{l}17 \\
(10 . \\
3 \%)\end{array}$ & 0.51 \\
\hline $\begin{array}{l}\text { Q13.There is } \\
\text { hardly any room } \\
\text { for unfair means }\end{array}$ & $\begin{array}{l}11 \\
(6.6 \\
\%)\end{array}$ & $\begin{array}{l}4 \\
(2.4 \\
\%)\end{array}$ & $\begin{array}{l}7 \\
(4.2 \%)\end{array}$ & $\begin{array}{l}13 \\
(7.8 \\
\%)\end{array}$ & $\begin{array}{l}4 \\
(2.4 \\
\%)\end{array}$ & $\begin{array}{l}9 \\
(5.4 \\
\%)\end{array}$ & $\begin{array}{l}27 \\
(16.3 \\
\%)\end{array}$ & $\begin{array}{l}14 \\
(8.4 \\
\%)\end{array}$ & $\begin{array}{l}12 \\
(7.2 \\
\%)\end{array}$ & $\begin{array}{l}26 \\
(15.7 \%)\end{array}$ & $\begin{array}{l}15 \\
(9.1 \%)\end{array}$ & $\begin{array}{l}23 \\
(14 \\
\%)\end{array}$ & 0.70 \\
\hline $\begin{array}{l}\text { Q14.I learn new } \\
\text { things while } \\
\text { preparing for } \\
\text { assessment }\end{array}$ & $\begin{array}{l}20 \\
(12.1 \\
\%)\end{array}$ & $\begin{array}{l}2 \\
(1.2 \\
\%)\end{array}$ & $\begin{array}{l}0 \\
(0 \%)\end{array}$ & $\begin{array}{l}18 \\
(11 \% \\
)\end{array}$ & $\begin{array}{l}2 \\
(1.2 \\
\%)\end{array}$ & $\begin{array}{l}6 \\
(3.6 \\
\%)\end{array}$ & $\begin{array}{l}42 \\
(25.4 \\
\%)\end{array}$ & $\begin{array}{l}7 \\
(4.2 \\
\%)\end{array}$ & $\begin{array}{l}4 \\
(2.4 \\
\%)\end{array}$ & $\begin{array}{l}58 \\
(35.1 \%)\end{array}$ & $\begin{array}{l}4 \\
(2.4 \%)\end{array}$ & $\begin{array}{l}2 \\
(1.2 \\
\%)\end{array}$ & 0.02 \\
\hline $\begin{array}{l}\text { Q15.I forgot } \\
\text { most of what I } \\
\text { learn after the } \\
\text { assessment }\end{array}$ & $\begin{array}{l}10 \\
(6 \%)\end{array}$ & $\begin{array}{l}7 \\
(4.2 \\
\%)\end{array}$ & $\begin{array}{l}5 \\
(3 \%)\end{array}$ & $\begin{array}{l}15 \\
(9.1 \\
\%)\end{array}$ & $\begin{array}{l}7 \\
(4.2 \\
\%)\end{array}$ & $\begin{array}{l}4 \\
(2.4 \\
\%)\end{array}$ & $\begin{array}{l}34 \\
(20.6 \\
\%)\end{array}$ & $\begin{array}{l}9 \\
(5.4 \\
\%)\end{array}$ & $\begin{array}{l}10 \\
(6 \%)\end{array}$ & $\begin{array}{l}35 \\
(21.2 \%)\end{array}$ & $\begin{array}{l}14 \\
(8.4 \%)\end{array}$ & $\begin{array}{l}14 \\
(8.4 \\
\%)\end{array}$ & 0.83 \\
\hline
\end{tabular}

\section{DISCUSSION}

It is well established fact that assessment not only drives learning but also influences students study habits markedly. ${ }^{11}$ Depending upon its role in the curriculum, assessment can be performed in many ways, like summative and formative etc. ${ }^{12}$ Ideally learning at university / professional level demands change in the style i.e. from superficial/ selective learning to deep learning. ${ }^{7}$ But usually learning style developed during the period of schooling take their toll in the professional level education in university as well. ${ }^{7}$ Our study results shown minimal change in the study habits of our dental students. They are still following the selective approach of studying near the assessment. But, our results were in agreement to the studies conducted by Anwar $\mathrm{M} \mathrm{et} \mathrm{al}^{7}$ as well as to Endstrom et al $^{12}$. They both reported the concept of studying selectively near the examinations. Students therefore should be assessed throughout the year so as to guide them 
through adequate feedback well timely to improve their learning. ${ }^{13}$ This infact was the concept to introduce formative assessment early in the program. ${ }^{7}$ Our study results further highlighted the consideration of formative assessment as a favored learning tool. This finding was in agreement to those of Figueroa $\mathrm{C}^{14}$ et al and Anwar $\mathrm{M}$ et al. ${ }^{7}$

Promising finding related to students was that the majority were taking formative assessment quite seriously and considering it useful for their preparation of summative assessment. Again this was also in agreement to other researchers. ${ }^{7}{ }^{15}$ Regarding frequency of formative assessment majority of students were considering it more. Reason might be that the trend of formative assessment was introduced in the recent past, so our students are not tuned to the frequent assessments. But this finding was also in partial agreement to those of Anwar M et al, whom study results shown more agreement to the existing frequency of formative assessment primarily by the senior compared to junior basic levelstudents. ${ }^{7}$

Most of students were agreed to the statement that there are too many assessments in a year. It could be a reason that at the end of a course assessment is usually viewed as a frightening obstacle so has some negative effects on learning as well. ${ }^{16}$ But the weightage of curriculum based assessment and correlation of the exam content with the learning objectives was considered appropriate. Similar results were also concluded by other researchers as well. ${ }^{7 \cdot 17}$

Majority of the students in this study agreed to the present number of MCQs in the exam. But most of them demanded inclusion of other types of MCQs besides one best type. It might be because one best MCQ needs significant brainstorming in order to solve the issue. Otherwise single best MCQs are more reliable and have high discriminating power compared to other type of MCQs.

18 Students in our study agreed to the inclusion of more number of SEQs in the exam. Our this finding was in contradiction to those of Anwar $\mathrm{M}$ et al, probably because of difference in curriculum being followed i.e. integrated compared to non integrated in our dental college. ${ }^{7}$ Majority of students were not agreed with the time allocation and quality of images/ stuff placed in the OSCE/ OSPE stations. This was the finding in common by another researcher who then advised to sort the issue by pilot testing of each station before examination. ${ }^{7}$ Student's remarks were quite positive about impartial invigilation and lack of unfair means about our summative assessment, but they claim to forget most of what they have learnt after assessment.

\section{CONCLUSION}

This study confirms that study habits at the time of admission to medical school are difficult to change but can be modified. It also confirms that students like formative assessment mainly because of feedback as it helps them in learning as well as preparing for summative assessment. Summative assessment, on the other hand, was more satisfactory for the students except that they opposed inclusion of more methods of assessment.

\section{REFERENCES}

1. Linquanti R W. Supporting Formative Assessment for Deeper Learning: A Primer for Policymakers. http://www.ccsso.org/Documents/Supporting Formative Assessment for Deeper Learning.pdf. Published 2014. Accessed August 12, 2016.

2. Wiliam D, Lee $C$, Harrison $C B P$. Teachers developing assessment for learning: impact on students achievement. Assess Educ Policy Pract. 2004;11(1):49-65. 
3. Black. P WD. Assessment and classroom learning. Assess Educ. 1998;5(1):7-74.

4. Shepard LA. Commentary: Evaluating the validity of formative and interim assessment. Educ Meas Issues Pract. 2009;28(3):32-37.

5. Morgan Jones RL, Wade R RJ. The motivation to learn:efficacy and relevance of the Oswestry postgraduate orthopaedic training programme. Ann R Coll Surg Engl. 1998;80:271-273.

6. Al- Kadri HM,Al-Moamery MMS, Magzoub ME, Roberts C VC. Students's Perceptions of the impact of assessment to learning: a comparision between two medical schools with similar curricula. Int $J$ Med Educ. 2011;2:44-52.

7. Anwar M HF. Students's Perception Towards Formative and Summative Assessment: A Single Institution Study. JIIMC. 2016;11(1):35-40.

8. McManus IC, Richards $P$, Winder BC SK. Clinical experiance, performance in final examinations, and learning styles in medical students: prospective study. BMJ. 1998;316:345-350.

9. Marton F SR. On qualitative differences in learning: II outcome as a function of the learners conception of the task. Br J Educ Psychol. 1976;46(1):4-11.

10. Lonka K L-YS. Epistemologies conception of learning, and study practices in medicine and psychology. High Educ. 1996;31:5-24.

11. Gibbs G SC. Conditions under which supportsstudents learning. Learn Teach High Educ. 2004;1(1):3-31.

12. Edstrom DW, Wilhemsson-Macleod N, Berggren M, Josephson A WC. A Phenomenographic study of students' conception of learning for a written examination. Int J Med Educ. 2015;6:40-46.

13. Ghiatau R, Diac G C V. Interaction between summative and formative in higher education assessment: Students's Perception. Pocedia Soc Behav Sci. 2011;11:220-224.

14. Figueroa C, Huidobro B, Wright AC, Riquelme A MR. Perceptions of medical students about formative assessments during clinical courses. Rev Med Chil. 2014;142:1193-1199.

15. Jain V A V. Use of Formative assessment as an educational tool. Use Form Assess as an Educ tool. 2012;24:3-4.

16. Baerheim A ME. Medical students proposing questions for their own written final examination: evaluation of an educational project. Med Educ. 2003;37:734-738.

17. Sadia S, Sultana S FW. OSCE as an Assessment Tool:Perceptions of Undergraduate Medical Students. Anaesth Pain Intensive Care. 2009;13:65-67.

18. an LT MAJ; FFEB. Introduction of single best answer questions as test of knowledge in the final examination for fellowship of the Royal College of Radiologists in Clinical Oncology. $R$ Coll Radiol. 2008;20(18):571-576.

LICENSE: JGMDS publishes its articles under a Creative Commons Attribution Non-Commercial Share-Alike license (CC-BY-NC-SA 4.0). COPYRIGHTS: Authors retain the rights without any restrictions to freely download, print, share and disseminate the article for any lawful purpose. It includes scholarly networks such as Research Gate, Google Scholar, LinkedIn, Academia.edu, Twitter, and other academic or professional networking sites. 